\title{
Lattice Boltzmann Simulation of Mixed Convection in a Driven Cavity Packed with Porous Medium
}

\author{
Zhenhua Chai ${ }^{1}$, Zhaoli $\mathrm{Guo}^{1}$, and Baochang $\mathrm{Shi}^{2}{ }^{2 \star}$ \\ ${ }^{1}$ State Key Laboratory of Coal Combustion, \\ Huazhong University of Science and Technology, 430074 Wuhan P.R. China \\ hustczh@126.com, \\ zlguo@mail.hust.edu.cn \\ 2 Department of Mathematics, \\ Huazhong University of Science and Technology, 430074 Wuhan P.R. China \\ sbchust@126.com
}

\begin{abstract}
The problem of mixed convection in a driven cavity packed with porous medium is studied with lattice Boltzmann method. A lattice Boltzmann model for incompressible flow in porous media and another thermal lattice Boltzmann model for solving the energy equation are proposed based on the generalized volume-averaged flow model. The present models have been validated by simulating mixed convection in a driven cavity (without porous medium) and it is found that the numerical results predicted by present models are in good agreement with available data reported in previous studies. Extensive parametric studies on mixed convection in a driven cavity filled with porous medium are carried out for various values of Reynolds number, Richardson number and Darcy number. It is found that the flow and temperature patterns change greatly with variations of these parameters.
\end{abstract}

Keywords: Lattice Boltzmann method; Mixed convection; Porous medium.

\section{Introduction}

Fluid flow and heat transfer in a driven cavity have recently received increasing attention because of its wide applications in engineering and science [12]. Some of these applications include oil extraction, cooling of electronic devices and heat transfer improvement in heat exchanger devices [3. From a practical point of view, the research on mixed convection in a driven cavity packed with porous medium is motivated by its wide applications in engineering such as petroleum reservoirs, building thermal insulation, chemical catalytic reactors, heat exchangers, solar power collectors, packed-bed catalytic reactors, nuclear energy

\footnotetext{
^ Corresponding author.
} 
systems and so on 34. These important applications have led to extensive investigations in this area 35617 .

In this paper, the problem of mixed convection in a driven cavity packed with porous medium is studied with the lattice Boltzmann method (LBM). The aim of the present study is to examine the effects of Reynolds number $(R e)$, Richardson number $(R i)$ and Darcy number $(D a)$ on characteristics of the flow and temperature fields. The numerical results in present work indicate that the flow and temperature patterns change greatly with variations of the parameters mentioned above. Furthermore, as these parameters are varied in a wide range, some new phenomena is observed.

\section{Numerical Method: The Lattice Boltzmann Method}

In the past two decades, the LBM achieved great success in simulating complex fluid flows and transport phenomena since its emergence 8 . In the present work, the LBM is extended to study mixed convection in a driven cavity filled with porous medium.

The dimensionless generalized volume-averaged Navier-Stokes equations and energy equation are written as 910.

$$
\begin{aligned}
\nabla \cdot \mathbf{u} & =0, \\
\frac{\partial \mathbf{u}}{\partial t}+\mathbf{u} \cdot \nabla\left(\frac{\mathbf{u}}{\epsilon}\right) & =-\nabla(\epsilon p)+\frac{1}{R e_{e}} \nabla^{2} \mathbf{u}+\mathbf{F}, \\
\sigma \frac{\partial T}{\partial t}+\mathbf{u} \cdot \nabla T & =\frac{1}{\operatorname{Pr} R e} \nabla^{2} T,
\end{aligned}
$$

where $\mathbf{u}$ and $p$ are the volume-averaged velocity and pressure, respectively; $\epsilon$ is the porosity of the medium, $R e_{e}$ is the effective Reynolds number, $\operatorname{Pr}$ is the Prandtl number, $\sigma=\epsilon+(1-\epsilon) \rho_{s} c_{p s} / \rho_{f} c_{p f}$ represents the ratio between the heat capacities of the solid and fluid phases, with $\rho_{s}\left(\rho_{f}\right)$ and $c_{p s}\left(c_{p f}\right)$ being the density and capacity of the solid (fluid) phase, respectively. $\mathbf{F}=-\frac{\epsilon}{D a R e} \mathbf{u}-$ $\frac{\epsilon F_{\epsilon}}{\sqrt{D a}}|\mathbf{u}| \mathbf{u}+\frac{\epsilon G r}{R e_{e}^{2}} \mathbf{k} T$, where $D a$ is the Darcy number, Re is the Reynolds number, which is assumed to equal $R e_{e}$ in the present work, we would like to point out that this assumption is widely used in engineering; $G r$ is the Grashof number, for simplicity, the Richardson number $(R i)$ is introduced, and defined as $\frac{G r}{R e_{e}^{2}}, \mathbf{k}$ is unit vector in the $y$-direction, $F_{\epsilon}$ is geometric function, and defined as 11 .

$$
F_{\epsilon}=\frac{1.75}{\sqrt{150 \times \epsilon^{3}}}
$$

The evolution equations of the single particle density distribution and temperature distribution can be written as [10]12

$$
\begin{aligned}
& f_{i}\left(\mathbf{x}+c_{i} \delta t, t+\delta t\right)-f_{i}(\mathbf{x}, t)=-\frac{1}{\tau_{f}}\left[f_{i}(\mathbf{x}, t)-f_{i}^{(e q)}(\mathbf{x}, t)\right]+\delta t F_{i}, \\
& g_{i}\left(\mathbf{x}+c_{i} \delta t, t+\delta t\right)-g_{i}(\mathbf{x}, t)=-\frac{1}{\tau_{g}}\left[g_{i}(\mathbf{x}, t)-g_{i}^{(e q)}(\mathbf{x}, t)\right]
\end{aligned}
$$


where $\delta t$ is time step, $f_{i}(\mathbf{x}, t)$ and $g_{i}(\mathbf{x}, t)$ are density distribution function and temperature distribution function, respectively, $\tau_{f}$ and $\tau_{g}$ are the dimensionless relaxation times. $f_{i}^{(e q)}(\mathbf{x}, t), g_{i}^{(e q)}(\mathbf{x}, t)$ are the equilibrium distribution functions corresponding to $f_{i}(\mathbf{x}, t)$ and $g_{i}(\mathbf{x}, t)$, which are given as

$$
\begin{aligned}
& f_{i}^{(e q)}(\mathbf{x}, t)=\omega_{i} \rho\left[1+\frac{c_{i} \cdot \mathbf{u}}{c_{s}^{2}}+\frac{\left(c_{i} \cdot \mathbf{u}\right)^{2}}{2 \epsilon c_{s}^{4}}-\frac{|\mathbf{u}|^{2}}{2 \epsilon c_{s}^{2}}\right], \\
& g_{i}^{(e q)}(\mathbf{x}, t)=\omega_{i} T\left[\sigma+\frac{c_{i} \cdot \mathbf{u}}{c_{s}^{2}}\right],
\end{aligned}
$$

where $w_{i}$ is weight coefficient and $c_{s}$ is the sound speed. Unless otherwise stated, $\sigma$ in Eqs. (3) and (7) is assumed to equal 1, and the same treatment can be found in Ref. 9]. In the present work, we choose two-dimensional nine-bit model where the discrete velocities are given as $c_{0}=(0,0), c_{i}=(\cos [(i-1) \pi / 2], \sin [(i-$ 1) $\pi / 2]) c(i=1-4), c_{i}=(\cos [(2 i-9) \pi / 4], \sin [(2 i-9) \pi / 4]) \sqrt{2} c(i=5-8)$, where $c=\delta x / \delta t, \delta x$ is lattice spacing. The sound speed in this D2Q9 model is given as $c_{s}=c / \sqrt{3}$, and the weights are $\omega_{0}=4 / 9, \omega_{i}=1 / 9(i=1-4), \omega_{i}=1 / 36$ $(i=5-8)$.

The forcing term $F_{i}$ in Eq. (41) is given as 12

$$
F_{i}=\omega_{i} \rho\left(1-\frac{1}{2 \tau}\right)\left[\frac{c_{i} \cdot F}{c_{s}^{2}}+\frac{\mathbf{u} F:\left(c_{i} c_{i}-c_{s}^{2} I\right)}{\epsilon c_{s}^{4}}\right] .
$$

The volume-averaged density and velocity are defined by

$$
\rho=\sum_{i=0}^{8} f_{i}(\mathbf{x}, t), \quad \mathbf{u}=\frac{\mathbf{v}}{d_{0}+\sqrt{d_{0}^{2}+d_{1} \mathbf{v}}},
$$

where $d_{0}, d_{1}$ and $\mathbf{v}$ are defined as $d_{0}=\frac{1}{2}\left(1+\frac{\delta t}{2} \frac{\epsilon}{D a R e}\right), d_{1}=\frac{\delta t}{2} \frac{\epsilon F_{\epsilon}}{\sqrt{D a}}, \rho \mathbf{v}=$ $\sum_{i=0}^{8} c_{i} f_{i}+\frac{\delta t}{2} \rho \frac{\epsilon G r}{R e_{e}} \mathbf{k} T$.

Through the Chapman-Enskog expansion, and in the incompressible limit, we can derive the macroscopic equations (1)-(3). The further detailed analysis on this procedure can be found in Refs. 1012. In addition, the boundary conditions should be treated carefully, here the non-equilibrium extrapolation scheme proposed in Ref. [13] is used, this is because this scheme has exhibited better numerical stability in numerical simulations.

\section{$3 \quad$ Numerical Results and Discussion}

The configuration described in present study is shown in Fig. 1. The geometry is a square cavity with a length $L=1$. The cavity is packed with a porous material that is homogeneous and isotropic.

The present models were validated by simulating mixed convection in a square cavity with a driving lid for $R e=100,400,1000$ and the corresponding $R i=$ $1 \times 10^{-2}, 6.25 \times 10^{-4}, 1 \times 10^{-4}$. The results were compared with available data 


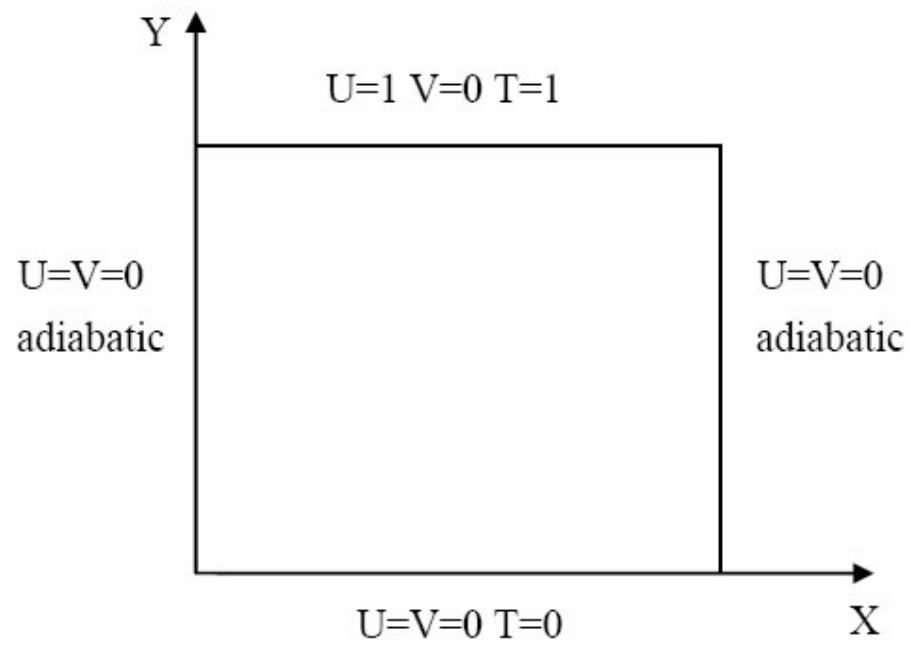

Fig. 1. The configuration of the problem under consideration

reported in previous studies in the absence of porous medium and heat generation for $\operatorname{Pr}=0.71$. It should be noted that the solution of mixed convection in the cavity without porous medium can be derived if $D a \rightarrow \infty, \epsilon \rightarrow 1$ and $\sigma=1$. As shown in Tables 1 and 2, the present numerical results are in good agreement with those reported in previous studies. In the following parts, we will focus on

Table 1. Comparisons of the average Nusselt number $(\overline{N u})$ at the top wall between the present work and that reported in previous studies

\begin{tabular}{cccccc}
\hline Re & Present Ref.[2 & Ref.[6] & FIDAP[6] & Ref.[5] \\
\hline 100 & 1.97 & 1.94 & 2.01 & 1.99 & 2.01 \\
400 & 4.03 & 3.84 & 3.91 & 4.02 & 3.91 \\
1000 & 6.56 & 6.33 & 6.33 & 6.47 & 6.33 \\
\hline
\end{tabular}

Table 2. Comparisons of the maximum and minimum values of the horizontal and vertical velocities at the center lines of the cavity between the present work and those reported in previous studies

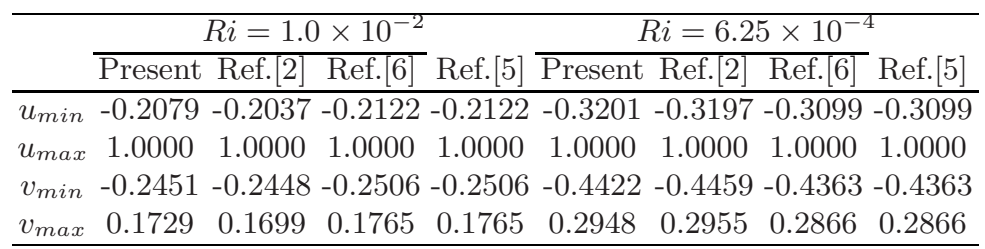


investigating the effect of variations of parameters, including $R e, R i$ and $D a$, on flow and temperature fields. The porosity of the medium is set to be 0.5 , and the Prandtl number is set to equal 0.71. Numerical simulations are carried out on a $257 \times 257$ lattice.

\subsection{Effect of the Reynolds Number (Re)}

The range of $R e$ is tested from 400 to 5000 under the condition of $R i=0.0001$ and $D a=0.01$. As shown in Fig. 2, the variation of Re has an important impact on flow and temperature fields. It is found that the qualitative character of flow is similar to the convectional lid-driven cavity flow of non-stratified fluid, a primary vortex is formed in center region of the cavity, and small vortexes are visible near the bottom corners with increasing $R e$. For temperature field, it is observed that there is a steep temperature gradient in the vertical direction near the bottom, and a weak temperature gradient in the center region. It is important that the convective region is enlarged with $R e$ increases.
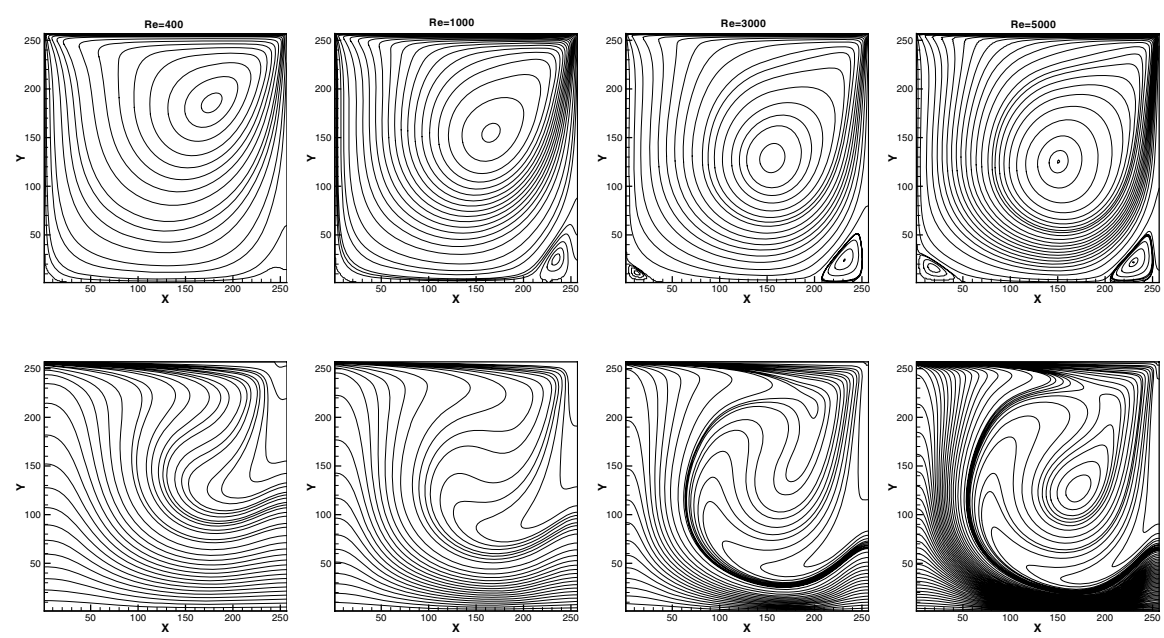

Fig. 2. The streamlines (top) and isothermals (bottom) for $R e=400,1000,3000,5000$

\subsection{Effect of the Richardson Number (Ri)}

The Richardson number is defined as the ratio of $G r / R e^{2}$, which provides a measure of the importance of buoyancy-driven natural convection relative to lid-driven cavity force convection. It reflects a dominant conduction mode as $R i \geq O(1)$, while it resembles similar driven cavity flow behavior for a nonstratified fluid if $R i \leq O(1)$ [26]. In the present work, $R i$ is varied in the range 



Fig. 3. The streamlines (top) and isothermals (bottom) for $R i=0.0001,0.01,1,10$

of $0.0001-10$ for $R e=100, D a=0.01$. As shown in Fig. 3, the flow and temperature fields change with the variations of $R i$. As $R i \leq 0.01$, the buoyancy effect is overwhelmed by the mechanical effect of the sliding lid, and only a primary vortex close to the top boundary is observed. As $R i$ increases to 1.0, beside the primary vortex mentioned above, another vortex is formed near the bottom corners. However, with increasing $R i$, the vortex near the bottom corner moves toward the geometric center, and finally comes to the center of the cavity as $R i$ is up to 10 . It is also shown that, as $R i \geq O(1)$, i.e., the effect of buoyancy is more prominent than mechanical effect of the sliding lid, the phenomena in driven cavity is more complex. The isothermals in Fig. 3 show the fact that heat transfer is mostly conductive in the middle and bottom parts of the cavity. The relative uniform temperature is only formed in a small region in the top portion of the cavity, where the mechanically induced convective activities are appreciable. However, it should be noted that this convective region is decreasing with increasing $R i$.

\subsection{Effect of the Darcy Number (Da)}

The $D a$ in the present work is varied in the range of $0.0001-0.1$ for $R i=0.0001$, $R e=100$. As shown in Fig. 4, the variation of $D a$ significantly affects the flow and temperature fields. It is obvious that the increase of $D a$ induces flow activity deeper into the cavity, which leads to more energy to be carried away from the sliding top wall toward bottom, and consequently, the convective region in the top portion of the cavity is enlarged. However, as $D a$ is decreased to 0.0001 , the primary vortex in the cavity is compelled to move toward left wall and a new 

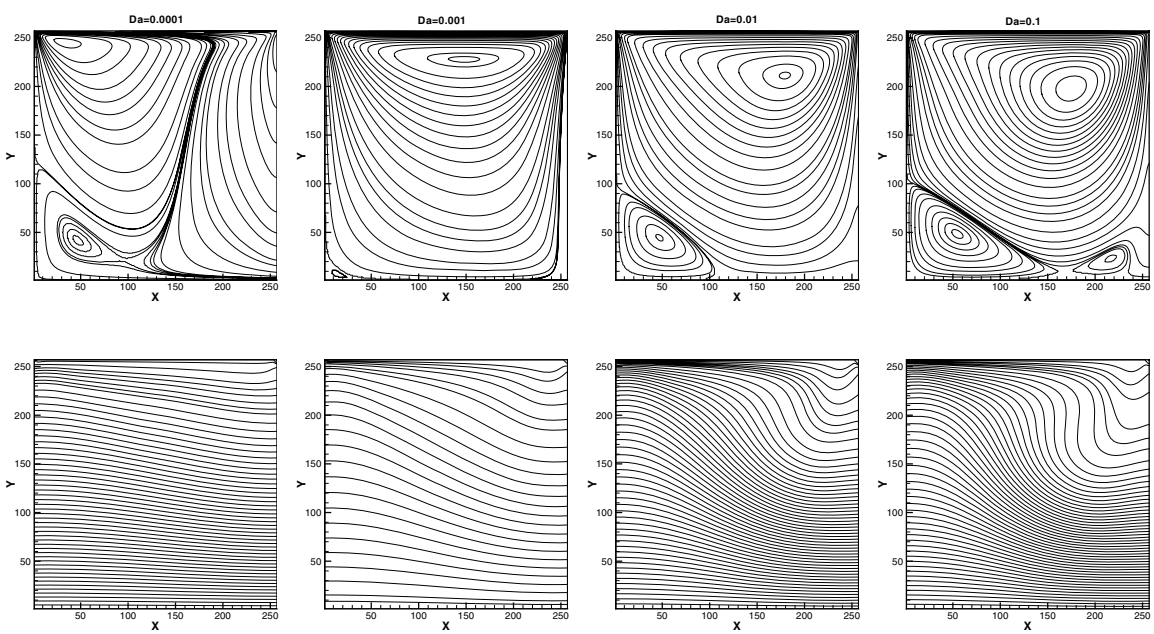

Fig. 4. The streamlines (top) and isothermals (bottom) for $D a=0.0001,0.001,0.01,0.1$

vortex near the bottom corner is formed. In fact, as $D a$ is small enough, the effect of the nonlinear term in Eq. (2) is prominent, which may induces some new phenomena as observed in present work. Finally, we would like to point out that the numerical results derived in this paper qualitatively agree well with those reported in Ref. 56].

\section{Conclusion}

In the present work, the problem of mixed convection in a driven cavity filled with porous medium is studied with LBM. The influence of the Reynolds number, Richardson number and Darcy number on the flow and temperature fields are investigated in detail. As these parameters are varied in a wide range, some new phenomena is observed. Through comparisons with the existing literature, it is found that the LBM can be used as an alternative approach to study this problem. Compared with traditional numerical methods, LBM offers flexibility, efficiency and outstanding amenability to parallelism when modelling complex flows, and thus it is more suitable for computation on parallel computers; but to derive the same accurate results, larger number of grid may be needed. A recent comparison between LBM and finite different method for simulating natural convection in porous media can be found in Ref. [14].

Acknowledgments. This work is supported by the National Basic Research Program of China (Grant No. 2006CB705804) and the National Science Foundation of China (Grant No. 50606012). 


\section{References}

1. Shankar, P.N., Deshpande, M.D.: Fluid mechanics in the driven cavity. Annu. Rev. Fluid Mech. 32 (2000) 93-136

2. Iwatsu, R., Hyun, J.M., Kuwahara, K.: Mixed convection in a driven cavity with a stable vertical temperature gradient. Int. J. Heat Mass Transfer 36 (1993) 16011608

3. Oztop, H.F.: Combined convection heat transfer in a porous lid-driven enclosure due to heater with finite length. Int. Commun. Heat Mass Transf. 33 (2006) 772 779

4. Vafai, K.: Convective flow and heat transfer in variable-porosity media. J. Fluid Mech. 147 (1984) 233-259

5. Khanafer, K.M., Chamkha, A.J.: Mixed convection flow in a lid-driven enclosure filled with a fluid-saturated porous medium. Int. J. Heat Mass Transfer 42 (1999) $2465-2481$

6. Al-Amiri, A.M.: Analysis of momentum and energy transfer in a lid-driven cavity filled with a porous medium. Int. J. Heat Mass Transfer 43 (2000) 3513-3527

7. Jue, T.C.: Analysis of flows driven by a torsionally-oscillatory lid in a fluidsaturated porous enclosure with thermal stable stratification. Int. J. Therm. Sci. 41 (2002) 795-804

8. Chen, S., Doolen, G.: Lattice Boltzmann method for fluid flow. Annu. Rev. Fluid Mech. 30 (1998) 329-364

9. Nithiarasu, P., Seetharamu, K.N., Sundararajan, T.: Natural convective heat transfer in a fluid saturated variable porosity medium. Int. J. Heat Mass Transfer 40 (1997) 3955-3967

10. Guo, Z., Zhao T.S.: A lattice Boltzmann model for convection heat transfer in porous media. Numerical Heat Transfer, Part B 47 (2005) 157-177

11. Ergun, S.: Fluid flow through packed columns. Chem. Eng. Prog. 48 (1952) 89-94

12. Guo, Z., Zhao, T.S.: Lattice Boltzmann model for incompressible flows through porous media. Phys. Rev. E 66 (2002) 036304

13. Guo, Z., Zheng, C., Shi, B.: Non-equilibrium extrapolation method for velocity and pressure boundary conditions in the lattice Boltzmann method. Chin. Phys. 11 (2002) 366-374

14. Seta, T., Takegoshi, E., Okui, K.: Lattice Boltzmann simulation of natural convection in porous media. Math. Comput. Simulat. 72 (2006) 195-200 